Revue européenne des sciences sociales

European Journal of Social Sciences

54-1 | 2016

Les usages sociaux des sciences du vivant

\title{
Yves GINGRAS, Sociologie des sciences
}

\author{
Mehdi K. Benslimane
}

\section{(2) OpenEdition}

\section{Journals}

Édition électronique

URL : http://journals.openedition.org/ress/3374

DOI : $10.4000 /$ ress.3374

ISBN : $1663-4446$

ISSN : 1663-4446

Éditeur

Librairie Droz

Édition imprimée

Date de publication : 15 mai 2016

Pagination : 273-276

ISSN : 0048-8046

Référence électronique

Mehdi K. Benslimane, "Yves GINGRAS, Sociologie des sciences 》, Revue européenne des sciences sociales [En ligne], 54-1 | 2016, mis en ligne le 15 mai 2016, consulté le 22 septembre 2020. URL : http://journals.openedition.org/ress/3374 ; DOI : https://doi.org/10.4000/ress.3374

Ce document a été généré automatiquement le 22 septembre 2020

(C) Librairie Droz 


\title{
Yves GINGRAS, Sociologie des sciences
}

\author{
Mehdi K. Benslimane
}

\section{RÉFÉRENCE}

Yves GINGRAS, 2013, Sociologie des sciences, Paris, PUF « Que sais-je ? », 128 p.

1 Professeur titulaire de la Chaire de recherche du Canada en Histoire et Sociologie des sciences à l'université du Québec à Montréal, et directeur scientifique de l'Observatoire des sciences et des technologies au sein de cette université, Yves Gingras est un connaisseur du sujet qu'il traite. L'auteur précise qu'il réalise non pas une étude sur les sociologues des sciences mais bien une sociologie des sciences qu'il définit comme l'étude des pratiques scientifiques institutionnalisées, et qu'il prend soin de distinguer, du reste, de l'histoire des sciences d'une part, et de la philosophie des sciences d'autre part; la première s'intéressant aux transformations temporelles qui caractérisent leur évolution, la seconde s'occupant de savoirs stabilisés méthodiquement obtenus et validés (p.3). Le cheminement suivi par Gingras, dans l'architecture constitutive des quatre chapitres du livre, procède d'une investigation qui va des «fondements socioculturels de la science " (chap. 1) aux "déterminants sociaux des connaissances scientifiques » (chap. 4) en passant par « les institutions de la science » (chap. 2) et par « le système social de la science » (chap. 3).

2 Traitant des fondements socioculturels des sciences, le premier chapitre se fait écho des travaux de sociologues qui ont « étudié les rapports entre les valeurs et les attitudes favorables ou hostiles au discours et aux pratiques scientifiques, et [qui] ont abordé la question des liens entre sciences, démocratie et expertise de même que celle du rôle des valeurs culturelles et religieuses [catholicisme, puritanisme, protestantisme, etc.] dans l'acceptation sociale de la science.» (p.11), principalement dans des disciplines comme l'astronomie et la biologie, lesquelles sont plus ou moins mobilisées dans les débats de société. Concernant les liens entre science et démocratie par exemple, Tocqueville observait déjà dans De la démocratie en Amérique «la tension entre les valeurs de la démocratie et celles qui peuvent contribuer à la promotion de la science 
désintéressée »(p.15). Son travail contient les prémices de la distinction connue aujourd'hui entre "science pure" et "science appliquée", dans la mesure où il y défendait la thèse en vertu de laquelle «les institutions démocratiques ont une tendance naturelle et inévitable à ne demander aux sciences que leurs applications immédiates et utiles » (p.16). Dans ce sens, Tocqueville craignait le déclin des sciences sous l'effet de l'affaissement de la recherche théorique - nécessaire au progrès - que seuls des dirigeants politiques éclairés, selon lui, peuvent promouvoir.

3 Le deuxième chapitre retrace le processus d'émergence des institutions de la science (le patronage, les universités, les académies, les laboratoires industriels et gouvernementaux) qui ont œuvré, chacune à sa manière et suivant les contextes, au développement des sciences modernes dans des lieux de production distincts. Il restitue en outre les phénomènes d'expansion géographique de la science et de formation des disciplines scientifiques.

4 Le troisième chapitre consacré au "système social de la science " est le plus "sociologique» des quatre. Gingras y défend la thèse de l'autonomie relative de la science par rapport aux autres sphères de la société, et y aborde quelques-uns des modèles descriptifs/explicatifs de la science moderne. Ainsi du modèle fonctionnaliste du sociologue américain Robert K. Merton qui établit les normes sociales nécessaires au bon fonctionnement du système social de la science: "universalisme ", " communalisme », " désintéressement » et « scepticisme organisé » (p. 54-55). Dans ce cadre, si la résistance aux nouvelles découvertes peut s'expliquer par cette dernière norme de scepticisme organisé, elle peut résider aussi, suivant le paradigme de Thomas Kuhn, dans un « conservatisme » sous-jacent à la culture (théories, concepts, méthodes) propre à une communauté scientifique, voire à une discipline déterminée (p. 71). La science est ainsi un champ de luttes caractérisé par la concurrence entre pairs pour la « reconnaissance scientifique » qui a, d'une part, plus de chance d'être attribuée à un chercheur appartenant à une université/institution de recherche reconnue, sur le plan international, qu'à un chercheur dépendant d'une institution marginale d'un pays « marginal» (p. 83) et qui, d'autre part, n'est pas sans liens avec la prédominance de la langue anglaise dans la mesure où une «forte tendance à l'homogénéisation linguistique [est] à l'œuvre dans un champ scientifique de plus en plus globalisé " (p. 84). Par ailleurs, des notions sont proposées comme celle de " querelle de priorité » laquelle a opposé plusieurs prix Nobel, à l'instar d'Albert Einstein et de Max Planck, sur la paternité de la découverte de la fameuse équation $\mathrm{E}=\mathrm{mc}^{2}$ (p. 62). L'accumulation des avantages par les chercheurs est théorisée notamment par "l'effet Saint Matthieu » qui, reprenant le principe biblique, suggère que la science moderne fonctionne de telle façon que les chercheurs les plus réputés et les plus visibles ont plus de chances de cumuler avantages et ressources que les chercheurs les moins en vue. Cet effet se double de surcroît d'un "effet Matilda", en référence à un texte de l'historienne américaine Margaret Rossiter dans lequel cette dernière pointe les discriminations/ inégalités dont sont victimes les femmes chercheurs par rapport à leurs compères masculins s'agissant de la reconnaissance scientifique (p. 82).

Le quatrième et dernier chapitre explore les courants sociologiques qui ont plus ou moins étudié les facteurs sociaux et culturels qui sinon déterminent du moins influencent l'élaboration de connaissances scientifiques. Ainsi du courant «sociologie de la connaissance » qui s'appuie sur les travaux de Karl Marx, du philosophe Max Scheler - le premier à avoir introduit cette expression - de Karl Mannheim, et surtout 
du médecin et bactériologiste polonais Ludwig Fleck qui a forgé les concepts de « collectif de pensée » et de «style de pensée » (p. 87), lesquels serviront plus tard dans les travaux de Kuhn. L'auteur montre les limites de ce courant qui en est resté à une approche schématique, typologique, statique et institutionnelle courante, du reste, dans les années 1920-1930.

La "sociologie marxiste des sciences" de l'entre-deux-guerres est inspirée par les travaux de Nicolas Boukharine, et surtout du texte majeur du physicien russe Boris Hessen sur Les Racines sociales et économiques des Principia de Newton dans lequel il défend la thèse de «la coïncidence parfaite entre les thèmes de la physique étudiés à cette époque [XVII ${ }^{e}$ siècle], nés des besoins de l'économie et de la technique, et le contenu central des Principia » (p. 91). Les travaux de Kuhn introduisent quant à eux les notions de "paradigme ", de "crise » et de "révolution scientifique ». En gros, la recherche fonctionne, selon les époques, suivant un cadre théorique, des méthodes, des techniques, etc. qui acquièrent une certaine légitimité auprès de la communauté scientifique. Les crises surviennent lorsque des «anomalies» dans l'explication des réalités sociales s'accumulent et dès lors que le paradigme explicatif en vogue devient inopérant. Les révolutions adviennent ainsi par le passage d'un paradigme à un autre sous l'effet de facteurs tout à la fois internes et externes. Le principal apport de ce courant est d'avoir établi "l'association entre une communauté (sociale) et un paradigme (cognitif) [qui] a ouvert la voie à une nouvelle réflexion sur les liens entre contexte social et contenu scientifique » (p.98) dépassant ainsi l'analyse mertonienne, laquelle se limitait au contexte institutionnel et social favorable ou non à la formation des connaissances. Quant à la "sociologie des intérêts", sous l'influence de Barry Barnes, elle s'attache à l'étude historique d'épisodes scientifiques datés, mettant en évidence le fait que, outre les intérêts sociaux, politiques et idéologiques, des intérêts cognitifs et techniques peuvent expliquer les choix ainsi que les objectifs scientifiques des chercheurs.

7 Par l'influence de David Bloor, «le programme fort " renvoie non pas à une théorie sociologique explicite qui serait rattachée à des concepts ou à des objets précis mais plutôt aux principes généraux nécessaires à la production d'une "bonne» théorie sociologique de la connaissance à travers l'énonciation de quatre principes: causal, impartial, symétrique et réflexif (p.105-107). Pour sa part, le courant dela «microsociologie des pratiques scientifiques» met en évidence l'importance des savoirs tacites dans la production de connaissances via des descriptions ethnographiques fines de la vie quotidienne (expérimentations, interactions entre chercheurs) au sein de laboratoires de recherche (p. 111).

8 Autour de Michel Callon et de Bruno Latour, l'approche de «l'acteur-réseau », inspirée autant par la terminologie sémiotique que par la démarche empirique et descriptive de l'ethnométhodologie, vise non pas à expliquer par le «social » ou par le «cognitif » la construction de connaissances scientifiques, comme le font la sociologie des intérêts, le programme fort ainsi que la théorie du champ scientifique, mais à mettre en lumière l'importance de "réseaux hétérogènes » liant humains et «non-humains » dans la formation des faits scientifiques. Utilisant des notions telles que l'intéressement, l'enrôlement ou l'inscription, cette approche tend à mettre les acteurs et les " actants " sur un pied d'égalité, d'une manière qui nie la différence de nature ontologique entre les deux, ce qu'on ne manque pas de lui reprocher. 
Un dernier courant enfin établit une distinction entre les « controverses scientifiques » et les «controverses publiques »; les premières étant l'œuvre d'experts, les secondes regroupant des savants et des profanes sur des sujets de société au sein de l'espace public via un ensemble de supports médiatiques.

10 L'auteur dresse en conclusion le constat final que la science contemporaine est devenue, depuis les années 1980, collective, internationale et surtout coûteuse. Si Gingras a le mérite de nous offrir une analyse nourrie par de nombreuses références bibliographiques datant d'époques et de courants scientifiques divers, le tout illustré par des encadrés et des figures, il aurait pu éviter néanmoins des énoncés aussi tranchés que le fait d'affirmer, par exemple, que «dans le premier cas [où l'auteur d'une publication scientifique originale n'est pas rétribué pour l'avoir écrit], il agit au sein de sa communauté, alors que dans le second [où le chercheur reçoit généralement des droits d'auteur pour écrire un manuel dans son domaine de recherche] il participe au marché de l'édition, lequel obéit aux règles du capitalisme marchand, ce qui a peu de valeur du point de vue strictement scientifique» (p. 64). Disant ceci, Gingras omet de remarquer que les chercheurs qui publient leurs travaux dans des revues scientifiques à comité de lecture se soumettent, à leur tour, dans le cadre du développement de leurs carrières, au marché des postes et du capital(isme) matériel, et par conséquent au marché de l'évaluation et de la reconnaissance professionnelle.

11 Il n'en demeure pas moins vrai que l'auteur nous fait plonger avec lui dans un monde scientifique passionnant, empli de découvertes, qui offre au lecteur-chercheur, quelque soit sa discipline, la possibilité de voir de nouvelles branches de la connaissance aussi bien dans les sciences de la nature que dans les sciences sociales, bien que les premières (mathématiques, physique, biomédecine, etc.) l'emportent sur les secondes, sans doute parce que l'auteur a été d'abord physicien avant de devenir sociologue. En outre, le livre permet aux chercheurs de comprendre et de prendre conscience d'un fonctionnement de "choses » qu'ils ont pris l'habitude de faire sans toujours savoir pourquoi et comment. Enfin, ce n'est pas seulement une sociologie des sciences que nous propose Gingras, mais également une socio-anthropologie historique des sciences en Europe et en Amérique du Nord depuis le XVII siècle. Et cela à rebours même du projet de l'auteur qui souhaitait pourtant inscrire son travail dans la seule discipline sociologique et dans une tradition marquée par une certaine influence - palpable surtout au niveau du troisième chapitre de l'ouvrage - de l'héritage de Bourdieu, laquelle se laisse voir notamment par le recours à la «théorie des champs » et les concepts que celle-ci mobilise.

\section{AUTEURS}

\section{MEHDI K. BENSLIMANE}

Université de Grenoble - PACTE (UMR 5194) 